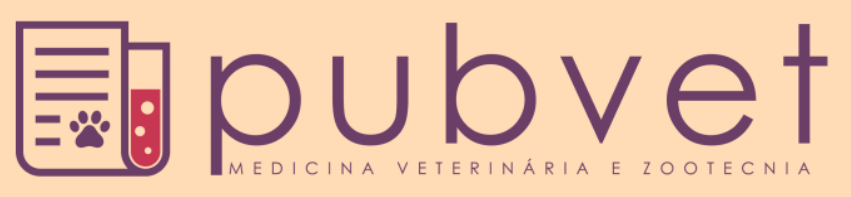

HTTP://DX.DOI.ORG/10.22256/PUBVET.V11N10.1028-1035

\title{
Urolitíase obstrutiva em ovinos: Revisão
}

Francisco das Chagas Cardoso Júnior ${ }^{1}$, Nair Chaves Barbosa da Silva1, Yanne Aciole da Silva $^{1}$, Alcir Martins Pereira ${ }^{1}$, Wendel de Sousa Mendonça ${ }^{1}$, Francisco Solano Feitosa Junior $^{2}$, Taciana Galba da Silva Tenório ${ }^{2 *}$

${ }^{1}$ Medico(a) Veterinário(a) pela Universidade Federal do Piauí - Teresina, Piauí, Brasil.

${ }^{2}$ Medica Veterinária, Professor (a) Dr(a) da Universidade Federal do Piauí, Departamento de Clínica e Cirurgia - Teresina, Piauí, Brasil.

${ }^{*}$ Autor para correspondência: E-mail: tacianagalba@yahoo.com.br

RESUMO. A urolitíase é um processo patológico caracterizado pela presença de cálculos ou concreções no sistema urinário. De etiologia complexa e multifatorial, caracterizada pela formação de urólitos, que se alojam no trato urinário e preocupam à medida que determinam sua obstrução, passando a ser denominado de urolitíase obstrutiva. Os primeiros sinais clínicos manifestados pelos animais com urolitíase obstrutiva estão relacionados à dor. Objetivou-se com esse trabalho uma atualização sobre o quadro patológico de urolitíase obstrutiva em ovinos, abordando desde seu histórico, etiologia, epidemiologia, patogenia, sinais clínicos, diagnóstico, diagnóstico diferencial, tratamento, controle e prevenção.

Palavras chave: obstrução, ovino, urolitíase

\section{Urolithiasis obstructive in sheep: Review}

ABSTRACT. Urolithiasis is a disease process characterized by the presence of calculi or concretions in the urinary tract. Complex and multifactorial etiology, characterized by the formation of uroliths, that lodge in the urinary tract and care as they determine their obstruction, becoming known as urolithiasis. The first clinical signs in the animals with urolithiasis are related to pain. The objective of this work an update on the pathological picture of obstructive urolithiasis in sheep from addressing its history, etiology, epidemiology, pathogenesis, clinical signs, diagnosis, differential diagnosis, treatment, control and prevention.

Keywords: obstruction, sheep, urolithiasis

\section{Urolitiasis obstructiva en ovinos: Revisión}

RESUMEN. La urolitiasis es un proceso patológico caracterizado por la presencia de cálculos o concreciones en el sistema urinario. De etiología compleja y multifactorial, caracterizada por la formación de urólitos, que se alojan en el tracto urinario y preocupan a medida que determinan su obstrucción, pasando a ser denominado de urolitiasis obstructiva. Los primeros signos clínicos manifestados por los animales con urolitiasis obstructiva están relacionados con el dolor. Este trabajo tuvo como objetivo abordar el tema de la urolitiasis obstructiva en ovinos desde su histórico, etiología, epidemiología, patogenia, manifestaciones clínicas, diagnósticos, diagnósticos diferencial, tratamiento, pronóstico, control y prevención.

Palabras clave: obstrucción, ovino, urolitiasis 


\section{Histórico}

Há trinta anos era impossível imaginar a criação de ovinos como um negócio organizado e rentável no Brasil. Na época a produção de pequenos ruminantes era vista como atividade secundária (Aquino Neto et al., 2007).

De 1991 a 2000, o abate mundial de pequenos ruminantes aumentou 7,5\%. Todavia a produtividade da caprino e ovinocultura de corte no Brasil ainda é baixa devido à predominância do sistema de produção extensivo, com alta dependência da vegetação nativa, utilização de raças não especializadas, uso de práticas rudimentares de manejo, assistência técnica deficitária, baixo nível de organização e de gestão da unidade produtiva (Guirro et al., 2013). Desde a década de 1970, o interesse pela produção de ovinos apresenta aumento gradativo no Brasil. Em 2011, o rebanho efetivo brasileiro de ovinos era de 17,6 milhões de cabeças, representando aumento de 1,6\% sobre o número registrado em 2010 (ANUALPEC, 2016).

A intensificação da produção, aliada à comercialização de animais de alto valor genético acarretou profundas alterações no manejo alimentar dos ovinos, desencadeando aumento da ocorrência de doenças nutricionais e metabólicas, e entre as principais se destaca a urolitíase obstrutiva (Guimarães et al., 2012).

Após o aparecimento dos sinais clínicos a reversão do quadro é difícil, podendo ser necessário tratamento cirúrgico quando, na grande maioria dos casos os animais tornam-se inaptos para a reprodução. Tais limitações econômicas, devido à prolongada terapia clínica e ao difícil acesso cirúrgico, frequentemente, fazem com que o animal seja descartado. Onde a adoção de práticas preventivas constitui a melhor medida para o controle desta doença (Ferreira et al., 2015).

Objetivou-se com esse trabalho uma atualização sobre o quadro patológico de urolitíase obstrutiva em ovinos, abordando desde seu histórico, etiologia, epidemiologia, patogenia, sinais clínicos, diagnóstico, diagnóstico diferencial, tratamento, controle e prevenção.

\section{Etiologia}

A urolitíase é um processo patológico caracterizado pela presença de cálculos ou concreções no sistema urinário (Riet-Correa et al., 2008). De etiologia complexa e multifatorial, caracterizada pela formação de urólitos, que se alojam no trato urinário e preocupam à medida que determinam sua obstrução, passando a ser denominado de urolitíase obstrutiva (Morais, 2012). Enfermidade esta possui muitas denominações, entre elas, cálculos urinários, pedras na bexiga, pedra nos rins, além de doença do trato urinário inferior (Aquino Neto et al., 2007). Existem diferentes tipos de cálculos, influências geográficas e sazonais, fatores dietéticos, ambientais, hormonais e doenças infecciosas do sistema urinário são descritas como fatores predisponentes à formação de urólitos (Ferreira et al., 2015). Cálculo urinário ou urolitíase forma-se quando solutos urinários inorgânicos ou orgânicos são precipitados para fora da solução. Os precipitados ocorrem como cristais ou "depósitos" amorfos. Os cálculos formam- se em um longo período de tempo pelo acúmulo gradual de precipitados ao redor de um núcleo (Radostits et al., 2010). Sendo essa uma doença nutricional que ocorre em consequência da precipitação de minerais ou substâncias orgânicas no trato urinário (Riet-Correa et al., 2001).

\section{Epidemiologia}

A distribuição da urolitíase obstrutiva em pequenos ruminantes é mundial. Esta doença foi relatada em todos os continentes e vários países com Austrália, Japão, Estados Unidos, Brasil, Índia e China (Morais, 2012). A urolitíase obstrutiva é caracterizada por formação de cálculos no trato urinário, que impedem o fluxo da urina. É uma doença amplamente discutida na literatura, acomete animais e humanos, e se relaciona com o manejo nutricional em todas as espécies. Ocorre com maior frequência e importância econômica em cordeiros, cabritos, garrotes, e animais de elite confinados (Ferreira, 2013).

Os urólitos são formados a partir da influência de fatores predisponentes, como, manejo intensivo dos animais, dieta excessivamente proteica ou com alto teor de fósforo, magnésio ou cálcio e ainda a ingestão de plantas com grande quantidade de oxalato ou sílica. Tais fatores não ocorrem de forma isolada, mas associados. Os machos são os mais afetados devido à anatomia da uretra peniana. $\mathrm{O}$ aumento da densidade urinária, a redução de água ingerida, desidratação, estase urinária, pH urinário alcalino, aumento de excreção mineral na urina, relacionada com a composição da dieta, a diminuição na concentração de coloide protetor da urina ou a descamação de células epiteliais da bexiga 
favorecem a precipitação de solutos que dão origem ao urólito (Dória et al., 2007, Radostits et al., 2010).

A urolitíase ocorre com maior frequência em sistemas de manejo intensivo, em que a dieta é formada basicamente de grãos. Esse alimento, de modo geral, tem elevado teor de fósforo e de magnésio, mas baixo teor de cálcio, dessa forma, a proporção de $\mathrm{Ca}$ e $\mathrm{P}$ varia de 1:4 a 1:6, enquanto que a relação ideal seria de $1: 1$ a $2: 1$. O desequilíbrio sanguíneo de Ca:P resulta em elevada excreção de fósforo pela urina, sendo importante fator para a gênese dos urólitos (Santarosa, 2015). Esta enfermidade é mais comum em machos castrados, com idade variando de 3 a 6 meses, devido à conformação da uretra, que difere em tamanho e diâmetro, quando comparada à de fêmeas (LIMA et al., 2011). A castração precoce pode induzir a hipoplásia uretral e peniana, com consequente diminuição do diâmetro da uretra (Santarosa, 2015).

A uretra dos machos é longa, tem menor calibre e apresenta ainda como os principais pontos de obstrução a flexura sigmoide, curvatura isquiática e processo uretral. Estas estruturas anatômicas facilitam a deposição de micro cálculos até a obstrução completa do fluxo urinário (Branchini, 2014).

A concentração da urina pela pouca ingestão de água pode causar urolitíase, e o problema é agravado em pastagens onde os animais não conhecem os acessos à água ou não há água de boa qualidade disponível. A dureza da água depende de íons presentes, principalmente, o cálcio e magnésio, na forma de carbonato. Autores correlacionaram positivamente à dureza da água consumida com a incidência da urolitíase (Sacco and Lopes, 2011). A estase urinária resultante de infecções e processos inflamatórios do trato urinário favorece o aparecimento de cálculos, pois o acúmulo de restos celulares altera o $\mathrm{pH}$ da urina, forma compostos salinos insolúveis, produz colóides estranhos à urina (pus e sangue), proporcionando a formação da matriz orgânica (Loretti et al., 2003).

$\mathrm{O} \mathrm{pH}$ urinário é outro fator muito importante que interfere na formação dos urólitos por afetar a solubilidade de alguns componentes presentes na urina (Radostits et al., 2010). A urina dos ruminantes é alcalina, seu $\mathrm{pH}$ varia em torno de 7,0 a 9,5. À medida que o pH aumenta, os colóides urinários perdem sua habilidade de comportaremse como um gel protetor, havendo precipitação de minerais (Aquino Neto et al., 2007). Quando o pH urinário aumenta um pouco e permanece próximo ao seu limite superior, o $\mathrm{pH}$ alcalino favorece a formação de cálculos de fosfato, estruvita e carbonato (Sun et al., 2010).

A concentração de fósforo na saliva é de 12 a 16 vezes maior do que o sangue, sendo esta de grande importância para o metabolismo do fósforo. Os ruminantes removem o fósforo do organismo pela sua excreção através da saliva, e daí para o rúmen e fezes. Normalmente, cerca de $60 \%$ do fósforo que chega ao rúmen é proveniente da saliva e os $40 \%$ restantes vêm da dieta. Dietas altas em concentrados e baixos em volumosos diminuem a formação de saliva, como resultado mais fósforo deve ser eliminado pelo rim e excretado na urina aumentando o risco de urolitíase (Riet-Correa et al., 2008, Morais, 2012).

Algumas plantas na pastagem podem conter até $6 \%$ de sílica. Cálculos provenientes deste mineral, geralmente aparecem em ovinos que se alimentam de plantas que crescem em solos arenosos ou que ingerem água que contem alto teor de sílica, o rim é a principal via de excreção do ácido silicico absorvido. Esse tipo de urolito é de pouca importância nos animais criados em regime intensivo (Bailey, 1981, Radostits et al., 2010). Riet-Correa et al. (2008) falam que o teor de sílica nas pastagens não é observado como causa de urolitíase no Nordeste. Animais que pastejam plantas que contenham oxalatos desenvolvem urólitos deste componente, devido à facilidade que o oxalato tem em ligar-se ao cálcio (Radostits et al., 2010).

A deficiência de vitamina $\mathrm{A}$ ou a administração de estrogênio são consideradas causa de excessiva queratinização do epitélio do sistema urinário, pois a descamação destas células queratinizadas servirá de núcleo para os sais precipitados (Aquino Neto et al., 2007, Riet-Correa et al., 2008). O uso de estrogênio além de aumentar a descamação do epitélio urinário, provoca também o aumento da produção de muco, favorecendo também a formação da matriz orgânica (Loretti et al., 2003).

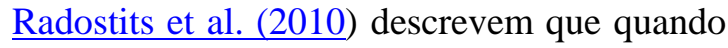
se utilizava estilbestrol como promotor do crescimento foi registrado taxas de mortalidade de $20 \%$ devido à urolitíase obstrutiva em animais que recebiam implante desse produto, quando comparados com a ausência de mortalidades no grupo-controle. Suspeitou-se que dietas com baixas concentrações de vitamina A fossem 
causadoras de urolitíase, porém, a deficiência de vitamina A não parece ser um dos principais fatores causadores.

A identificação de um animal com urolitíase no rebanho sugere que todos os machos do rebanho estão em risco, por conta da importância dos fatores dietéticos e ambientais em sua patogenia (Branchini, 2014).

\section{Patogenia}

$\mathrm{O}$ mecanismo fisiológico mais eficiente para excreção do excesso de minerais na corrente sanguínea é por meio da filtração renal. Este processo possibilita ao animal expulsar pelo trato urinário os minerais e conservar o balanço cátionaniônico normal (Araújo et al., 2011). A urolitíase obstrutiva desenvolve-se em ruminantes quando solutos urinários inorgânicos ou orgânicos são precipitados e depositam-se sobre uma matriz orgânica, que se solidificará transformando-se em urólito. A matriz ou núcleo é habitualmente um mucopolissacarídeo ou mucoproteína e podem consistir de leucócitos mortos, fibrina, restos celulares ou bactérias aglutinadas (Aquino Neto et al., 2007).

Três diferentes tipos de fatores atuam para a formação de cálculos: os que favorecem a precipitação das substâncias que se encontram em solução; os que favorecem a formação de uma matriz orgânica que atua como núcleo para a formação dos urólitos; os que favorecem a união e concreção dos precipitados e do núcleo agregando-os para formar os cálculos (RietCorrea et al., 2001).

Os locais mais comuns de obstrução incluem o processo uretral (apêndice vermiforme) e a flexura sigmoide distal, embora o trígono vesical, a bexiga e a pelve renal também sejam locais de grande acúmulo de cálculos (Ferreira, 2013).

\section{Sinais clínicos}

O quadro clínico pode variar dependendo da localização da obstrução. Se esta é completa ou parcial e da duração (Guimarães et al., 2012, Morais, 2012, Branchini, 2014). Os primeiros sinais clínicos manifestados pelos animais com urolitíase obstrutiva estão relacionados à dor, os animais apresentam: desconforto abdominal, escoiceamento do abdome, manoteio, balançar da cauda, decúbito intermitente com inquietação, anorexia, dificuldade de andar, marcha rígida, podendo estar presente, também, a exposição do pênis. Geralmente o animal faz esforço para urinar, adotando postura de micção, com contração espasmódica do pênis, sendo visível a movimentação do prepúcio. Pode haver grunhidos e bruxismo (Aquino Neto et al., 2007, Riet-Correa et al., 2008, Radostits et al., 2010, Ferreira et al., 2015, Santarosa, 2015, Branchini, 2014).

Quando a obstrução é total, a região prepucial está seca, um intenso precipitado de cristais normalmente é visível nos pelos prepuciais ou por dentro das coxas (Riet-Correa et al., 2008, Aquino Neto et al., 2007). À palpação retal digital nota-se aumento de sensibilidade, no local da obstrução, e pulsação da uretra, pode revelar bexiga repleta ou não palpável (Radostits et al., 2010, Aquino Neto et al., 2007, Branchini, 2014).

Os animais que desenvolvem urolitíase podem apresentar polaquiúria, disúria, hematúria, piúria e hipertermia (Riet-Correa et al., 2008, Radostits et al., 2010, Ferreira et al., 2015). Como sequela da obstrução do trato urinário podem ocorrer perfuração e ruptura uretral, constrição uretral, ruptura vesical, hidroureter, hidronefrose, prolapso retal, e raramente, ruptura do rim (Branchini, 2014). Quando há ruptura da bexiga o animal apresenta melhoria aparente pelo alívio da dor. Posteriormente, há extravasamento de urina para o tecido conjuntivo da parede abdominal ventral e prepúcio ou cavidade abdominal causando irritação do tecido, necrose, reabsorção de urina e uremia. A morte por uremia ocorre 2-3 dias após a ruptura.

\section{Diagnóstico}

O diagnóstico de urolitíase obstrutiva em pequenos ruminantes baseia-se principalmente no histórico, dados epidemiológicos, sinais clínicos. Porém, exames complementares como exames laboratoriais (urinálise, bioquímica clínica, e hemogramas), radiológicos e ultrassonográficos podem ajudar a elucidar o diagnóstico, e em alguns casos revelar a presença e composição dos urólitos (Radostits et al., 2010, Lima et al., 2012, Morais, 2012, Branchini, 2014, Ferreira et al., 2015).

A urinálise é composta de três fases: exame físico, químico, e análise microscópica do sedimento. Esse exame fornece informações preliminares no que diz respeito a distúrbios como hemorragia glomerular, hepatopatias, erros inatos do metabolismo e infecções do trato urinário. A aferição da densidade urinária ajuda na avaliação da função dos túbulos renais. Os resultados do exame físico da urina também podem ser 
utilizados para confirmar ou explicar achados do exame químico e microscópico (Radostits et al., 2010, Araújo et al., 2011, Morais, 2012, Ferreira, 2013, Ferreira et al., 2015).

A bioquímica sérica revela azotemia pós-renal, que se relaciona à estase urinária e consequente reabsorção desses compostos. Os níveis de creatinina plasmática refletem a taxa de filtração renal, de forma que os altos níveis indicam deficiência na funcionalidade renal. Os níveis séricos de ureia são influenciados pela nutrição, visto que a concentração sérica e excreção se elevam com o aumento do teor de proteína da dieta. Em dietas com baixos níveis de proteína bruta, a maioria da ureia é reciclada e muito pouco se perde na urina (Radostits et al., 2010, Ferreira et al., 2015, Santarosa, 2015).

Os exames de diagnóstico por imagem como radiografia, ultrassonografia, podem auxiliar no diagnóstico de alterações no trato urinário, além de confirmar a presença de urólitos nos rins, ureter, bexiga e uretra. Porém, a ultrassonografia tem sido o método de diagnóstico por imagem mais utilizada para a detecção da urolitíase, devido à sua capacidade de visualizar cálculos radioluscentes, enquanto a radiografia permite observar apenas os radiopacos. Além de ser de fácil acesso, baixo custo-operacional, boa sensibilidade e por não ser invasivo (Radostits et al., 2010, Lima et al., 2012, Ferreira et al., 2014).

\section{Diagnóstico diferencial}

$\mathrm{Na}$ obstrução uretral, as principais características são dor abdominal e anorexia, que deverão ser diferenciadas de outras enfermidades intestinais como obstrução intestinal, coccidiose, salmonelose e indigestão aguda (Radostits et al., 2010, Ferreira et al., 2015). O diagnóstico diferencial em casos de ruptura uretral é realizado entre abscessos subcutâneos, abscessos umbilicais, hérnias umbilicais e ventrais, hematomas e lesão prepucial (Radostits et al., 2010, Ferreira et al., 2015). Quando há ruptura de bexiga o abdome apresenta-se distendido e deve ser diferenciado de timpanismo ruminal, indigestão vagal e ascite. Com a evolução do quadro clínico e do estado urêmico, o animal apresenta depressão severa, anorexia e desidratação. Nesta situação, devem ser realizados exames neurológicos, para excluir suspeita de raiva (Radostits et al., 2010, Ferreira et al., 2015).

\section{Tratamento}

A urolitíase obstrutiva, em pequenos ruminantes, ainda é um problema de difícil tratamento, e, quando se consideram resultados em longo prazo o tratamento cirúrgico torna-se imprescindível (Ferreira et al., 2015). O tratamento utilizado depende do estágio da doença, da natureza e extensão dos cálculos presentes, da função do animal e, frequentemente, das questões financeiras (Ferreira, 2013). O animal com urolitíase obstrutiva deve ser tratado como uma emergência (Branchini, 2014, Santarosa, 2015). O tratamento medicamentoso deve ser tentado nos estágios iniciais da obstrução ou quando esta é parcial (Riet-Correa et al., 2001). O tratamento para a urolitíase obstrutiva em ovinos visa o restabelecimento do fluxo urinário e a correção dos desequilíbrios hídrico eletrolítico (Fraser et al., 1996, Aquino Neto et al., 2007). Existem dois tipos de tratamento para esta enfermidade, o conservativo e o cirúrgico, cada um deles apresentando vantagens e desvantagens, que devem ser detalhadamente expostas ao proprietário e adotadas pelo médico veterinário após consideração individual de cada caso clínico (Aquino Neto et al., 2007, Branchini, 2014). O tratamento conservativo inclui amputação do processo uretral, antibioticoterapia para evitar infecções bacterianas secundárias, administração de anti-espasmódicos, administração de relaxantes musculares, analgésicos, acidificantes urinários e reposição hidroeletrolítica (Riet-Correa et al., 2001, Aquino Neto et al., 2007, Branchini, 2014). A primeira tentativa no tratamento da urolitíase pode ser realizada com o uso de relaxantes musculares, sendo que o objetivo primário do atendimento ao paciente obstruído é restabelecer o fluxo urinário. A hioscina, associada à dipirona pode ser utilizada como relaxante e analgésico, enquanto a xilazina deve ser evitada pela ação diurética. A acepromazina é o sedativo de escolha, pois tem melhor efeito antiespasmódico na uretra (Ferreira, 2013, Santarosa, 2015). Caso a obstrução não seja desfeita dentro de 12 a 18 horas depois da primeira aplicação, outro tratamento deve ser adotado (Aquino Neto et al., 2007).

Dos métodos utilizados em terapia na Medicina Natural, a fitoterapia é, sem dúvida, um dos mais antigos. Trevisan et al., (2009), descreveram um tratamento alternativo que se demonstrou eficaz para um ovino acometido por urolitíase pela da utilização do extrato fitoterápico DC 300, elaborado a partir de plantas da família Caryophyllaceae. O extrato em questão se 
demonstrou um excelente candidato para o desenvolvimento de um futuro fitoterápico, não tendo efeito tóxico, de uso seguro no tratamento de animais domésticos e é eficiente na terapia de urolitíase obstrutiva ovina. Quando o tratamento conservativo não é eficiente para desfazer a obstrução, a intervenção cirúrgica faz-se necessária, devendo-se levar em consideração a localização da obstrução, integridade da uretra ou bexiga e o valor e pretensão de uso do animal (Aquino Neto et al., 2007). A maioria dos tratamentos cirúrgicos tem por finalidade salvaguardar a vida do animal até o momento do abate (Meneses et al., 2007).

Diferentes técnicas cirúrgicas são descritas para o tratamento desses casos de urolitíase, incluindo amputação do processo uretral, transposição peniana, penectomia, uretrostomia perineal, fistulização da bexiga e a cistotomia (Radostits et al., 2010, Riet-Correa et al., 2001, Dória et al., 2007, Meneses et al., 2007, Sousa et al., 2011). A amputação do processo uretral é uma técnica de sucesso em praticamente metade dos casos de urolitíase em pequenos ruminantes, e a mais usada. A amputação do processo deve ser feita antes da sondagem, pois se consegue restaurar o fluxo de urina em cerca de $70 \%$ dos pacientes, quando a obstrução for próxima à glande. Esta técnica associada a fármacos pode promover alívio temporário, em no máximo 36 horas (Ferreira et al., 2015). Entretanto, a taxa de recorrência é alta, com 80 a $90 \%$ de casos de reobstrução no intervalo de horas a dias (Dória et al., 2007). Além disso, deve-se corrigir o desequilíbrio hidroeletrolítico do animal após desobstrução. Recomenda-se associar o uso de antibióticos de amplo espectro de ação de forma profilática, e anti-inflamatórios não esteroides, importante para controle da dor e redução dos sinais de inflamação (Ferreira, 2013, Ferreira et al., 2015). Devido às dificuldades associadas aos tratamentos e as complicações da urolitíase, podese ter como resultar a inutilização do macho para a reprodução e na perda de valores genéticos e econômicos, sendo assim a prevenção é fundamental (Branchini, 2014).

\section{Controle e prevenção}

Vários agentes e procedimentos de manejo são recomendados para a prevenção da urolitíase em ovinos e novilhos de engorda. Para isso, deve-se conhecer a composição química dos urólitos e corrigir todos os possíveis fatores que podem estar relacionados com a sua formação. (Radostits et al.,
2010, Riet-Correa et al., 2008, Santarosa, 2015). Primeiro, e provavelmente mais importante, a dieta deve conter um equilíbrio adequado de minerais, principalmente cálcio e fósforo, para evitar a precipitação do excesso de fósforo na urina. Que deve conter uma proporção de 1,5:1 ou 2:1 a mais recomendada (Riet-Correa et al., 2008, Radostits et al., 2010, Branchini, 2014). A ingestão de correta e de qualidade de água é fundamental para promover diurese e evitar concentração urinária de minerais. Os proprietários devem ser instruídos a respeito da importância deste fator para os animais mais predispostos a manifestar urolitíase. Deste modo, grandes lotes de animais criados em sistema extensivo devem ser providos de várias fontes de água para facilitar o acesso, já que dificilmente um ovino se afastaria do rebanho em busca de água (Aquino Neto et al., 2007, Ferreira, 2013).

Animais confinados, que estiverem com deficiência de vitamina $A$, pode ser suplementados, o que evita a descamação excessiva do epitélio das vias urinárias (Ferreira, 2013). No entanto, Lindley et al. (1953) não observaram interferência da vitamina A na ocorrência de urolitíase em ovinos. Radostits et al. (2010) citaram que um adequado consumo de vitamina A deve ser assegurado durante os períodos de seca e quando os animais forem alimentados para engorda com ração que contenha grãos.

O adiamento da castração pode reduzir a incidência de urolitíase obstrutiva, por permitir maior dilatação da uretra, o que facilita a excreção de cálculos menores, porém, a melhora não é bastante significativa. Sabe-se, porém, que na rotina de confinamento de cordeiros não se adota esse procedimento, visto que os animais são abatidos precocemente (Radostits et al., 2010, Riet-Correa et al., 2008, Santarosa, 2015). A acidificação da urina é um dos métodos mais eficientes e baratos para a prevenção da urolitíase. Pode ser realizada com a administração de dieta aniônica (Ferreira et al., 2014). O cloreto de amônio pode ser utilizado na prevenção de urólitos de estruvita e fosfato de cálcio, aumentando a solubilidade destes, que são preferencialmente formados por $\mathrm{pH}$ alcalino (Branchini, 2014, Santarosa, 2015). Pode ser utilizado na dieta total, na proporção de $0,5 \%$ a $1,0 \%$ ou $2 \%$ do concentrado, ou em doses individuais de 5 a 10g/animal/dia (Aquino Neto et al., 2007, Ferreira et al., 2014). Faial et al. (2014) descrevem que administração de cloreto de sódio 
a 4\% na ração é utilizada para o controle da urolitíase em carneiros castrados confinados e rações com até $13 \%$ de cloreto de sódio são fornecidas a ovelhas por longos períodos sem efeitos deletérios aparentes, desde que haja acesso livre à água, que irá promover uma maior diluição da urina. A adição gradual de cloreto de sódio até o nível de 3 a $5 \%$ da ingestão de matéria seca reduz a incidência de urolitíase (Aquino Neto et al., 2007, Riet-Correa et al., 2008).

A vitamina $\mathrm{C}$ é citada pela literatura como tendo resultados satisfatórios quando administrados de 3 a 4mg/kg/dia (Ferreira, 2013). No entanto Ferreira (2013) comprovou que o pH urinário em ovinos não permaneceu constantemente ácido, como o uso do mesmo. Aumentar o teor de fibras da dieta promove um tempo maior de ruminação. $\mathrm{O}$ atrito das papilas com as fibras estimula o sistema nervoso a aumentar a produção de saliva e secreção de fósforo. Este processo diminui a excreção de fósforo na urina e também seus valores séricos (Branchini, 2014). Em trabalho realizado por Minervino et al. (2014) foi demonstrado que a dieta com polpa cítrica estimula a ruminação dos ovinos, o que produziu maior quantidade de saliva, e consequentemente de $\mathrm{P}$, excretada para o conteúdo ruminal, evitando-se a necessidade de excreção deste mineral pela urina, bem como o aumento do risco da formação de cálculos.

\section{Conclusão}

A urolitíase obstrutiva é uma enfermidade de cuidados principalmente ligados ao regime alimentar a que os animais são submetidos. Onde se diagnostica no início de seu quadro, tem boas chances de correção, em contrapartida, a maioria dos animais acometidos por ela é destinada ao abate, tendo sua vida reprodutiva interrompida, acarretando diversas perdas genéticas e econômicas.

\section{Referências Bibliográficas}

ANUALPEC. 2016. Anuário da Pecuária Brasileira, 20th edn. Instituto FNP, São Paulo, SP, Brasil.

Aquino Neto, H. M., Facury Filho, E. J., Carvalho, A. Ú., Souza, F. A. \& Rezende, L. J. 2007. Urolitíase obstrutiva em ovinos: revisão de literatura. Veterinária em Foco, 4, 191-202.

Araújo, P. B., Teixeira, M. N., Coleho, M. C. O. C. \& Alencar, S. P. 2011. Urinálise como instrumento auxiliar no diagnóstico de enfermidades em pequenos ruminantes. Revista de Medicina Veterinária, 3, 30-38.

Bailey, C. B. 1981. Silica metabolism and silica urolithiasis in ruminants: a review. Canadian Journal of Animal Science, 61, 219-235.

Branchini, N. S. 2014. Estudo da influência alimentar na etiologia da urolitíase em cordeiros. Faculdade de Medicina $e$ Veterináira. Universidade Estadual Paulista Júlio de Mesquita Filho, Jaboticabal.

Dória, R. G. S., Canola, P. A., Dias, D. P. M., Pereira, R. N. \& Valadão, C. A. A. 2007. Técnicas cirúrgicas para urolitíase obstrutiva em pequenos ruminantes: relato de casos. Arquivo Brasileiro de Medicina Veterinária e Zootecnia, 59, 1425-1432.

Faial, K. C. F., Oliveira, C. M. C., Duarte, M. D., Bomjardim, H. d. A., Barbosa Neto, J. D., Lima, D. H. S., Silva, N. \& Bezerra Júnior, P. S. 2014. Surto de intoxicação por sal em ovinos no estado do Pará. Pesquisa Veterinaria Brasileira, 34, 1061-1068.

Ferreira, D. O. L. 2013. Modelo experimental de urolitíase em ovinos: estudo clinico, laboratorial e hemogasométrico. Faculdade de Medicina Vetrinária e Zootecncia. Universidade Estadual Paulista, Botucatu.

Ferreira, D. O. L., Santarosa, B. P., Amorim, R. M., Chiacchio, S. B. \& Gonçalves, R. C. 2015. Urolitíase obstrutiva em ovinos. Veterinária e Zootecnia, 22, 183-197.

Ferreira, D. O. L., Santarosa, B. P., Sacco, S. R., Dias, A., Amorim, R. M., Chiacchio, S. B., Lisbôa, J. A. N. \& Gonçalves, R. C. 2014. Efeito da suplementação de cloreto de amônio sobre os equilíbrios eletrolítico e ácido-básico e o pH urinário de ovinos confinados. Pesquisa Veterinária Brasileira, 34, 797-804.

Fraser, C. M., Bergeron, J. A., Mays, A. \& Aiello, S. A. 1996. Manual Merck de Veterinária: um manual de diagnóstico, tratamento, prevenção $e$ controle de doenças para o veterinário. Roca.

Guimarães, J. A., Mendonça, C. L., Sousa Guaraná, E. L., Dantas, A. C., Azevêdo Costa, N., Câmara, A. C. L., Farias, C. C. \& Afonso, J. A. B. 2012. Estudo retrospectivo de 66 casos de urolitíase obstrutiva em ovinos. Pesquisa Veterinária Brasileira, 32, 824-830.

Guirro, E. C. B. P., Sponchiado, D., Keller, D., Gusmão, M. A. \& Sponchiado, R. 2013. Perfil das propriedades produtoras de pequenos 
ruminantes de corte de Palotina/PR. Arquivos de Ciências Veterinárias e Zoologia da UNIPAR, 15, 13-17.

Lima, A. G., Costa, L. A. V. S., Silva, P. C. A. R., Barione, G., Baiotto, G. C. \& Costa, F. S. 2012. Diagnóstico ultrassonográfico de divertículo vesical adquirido em ovino com urolitíase obstrutiva-relato de caso. Revista de Medicina Veterinária, 5, 19-22.

Lindley, C. E., Taysom, E. D., Ham, W. \& Schneider, B. H. 1953. Urinary calculi in sheep. Journal of Animal Science, 12, 704-714.

Loretti, A. P., Oliveira, L. O., Cruz, C. E. F. \& Driemeier, D. 2003. Clinical and pathological study of an outbreak of obstructive urolithiasis in feedlot cattle in southern Brazil. Pesquisa Veterinária Brasileira, 23, 61-64.

Meneses, D. C. R., Júnior, F. S. F., Quessada, A. M., Silva, S. V., Pereira, H. M., Neiva, C. B., Guimarães, A. L. S. \& Oliveira, L. L. 2007. Fistulização da bexiga para tratamento de urolitíase obstrutiva em caprinos. Acta Veterinaria Brasilica, 1, 89-93.

Minervino, A. H. H., Araujo, C. A. S. C., Kaminishikawahara, C. M., Soares, F. B., Rodrigues, F. A. M. L., dos Reis, L. F., Oliveira, F. L. C., Junior, R. A. B., Mori, C. S. \& Ortolani, E. L. 2014. Influência de diferentes dietas com alto teor de concentrados sobre parâmetros ruminais, bioquímicos e urinários de ovinos. Brazilian Journal of Veterinary Research and Animal Science, 51, 30-36.

Morais, M. 2012. Estudio clínico epidemiológico da urolitíase obstructiva em caprinos e ovinos. Departamento de Medicina |Veterinária. Universidad Federal Rural de Permambuco, Permambuco.

Radostits, O. M., Gay, C. C., Blood, D. C., Hinchcliff, K. W. \& McKenzie, R. A. 2010. Clínica Veterinária: um tratado de doenças dos bovinos, ovinos, suínos, caprinos $e$ eqüinos. Guanabara Koogan, Rio de Janeiro.

Riet-Correa, F., Méndez, F., Carmen Schild, M., Riet-Correa, A. L. F., Schild, A. L. \& Carmen, M. M. 2001. Doenças de ruminantes e eqüinos. Varela, Sãp Paulo.

Riet-Correa, F., Simões, S. V. D. \& Vasconcelos, J. S. 2008. Urolitíase em caprinos e ovinos. Pesquisa Veterinaria Brasileira, 28, 319-322.

Sacco, S. R. \& Lopes, R. S. 2011. Urolitíase: estudo comparativo em bovinos Guzerá oriundos de propriedades com e sem o problema. Pesquisa Veterinária Brasileira, 31, 206-212.

Santarosa, B. P. 2015. Avaliação clínica, laboratorial e anatopatológica do sistema urinário de ovinos confinados com e sem suplementação de cloreto de amônio. Faculdade de Medicina Veterinária e Zootecnia. Universidade Estadual Paulista Júlio Mesquita Filho, Botucatu.

Sousa, A. A. R., Soares Nunes, V. H., Almeida de Oliveira, R., Sousa, S., Abreu, A., Cabral Teixeira, W., Coelho, C. \& Oliveira, M. C. 2011. Urolitíase obstrutiva em ovino. Acta Scientiae Veterinariae, 39, 1-4.

Sun, W.-D., Zhang, K.-C., Wang, J.-Y. \& Wang, X.-L. 2010. The chemical composition and ultrastructure of uroliths in Boer goats. The Veterinary Journal, 186, 70-75.

Article History:

Received 20 February 2017

Accepted 27 March 2017

Available on line 20 July 2017

License information: This is an open-access article distributed under the terms of the Creative Commons Attribution License 4.0, which permits unrestricted use, distribution, and reproduction in any medium, provided the original work is properly cited. 